Pak. J. Agri. Sci., Vol. 53(3), 487-494; 2016

ISSN (Print) 0552-9034, ISSN (Online) 2076-0906

DOI:10.21162/PAKJAS/16.3386

http://www.pakjas.com.pk

\title{
PROMOTER CLONING AND EXPRESSION ANALYSIS OF TRANSCRIPTION FACTOR GENE GMMYB92 IN SOYBEAN (Glycine max. L)
}

\author{
Huang Yihong1, ${ }^{1,}$ Xu Zhaolong, ${ }^{1,}$, Xu Ling1, Wei Peipei ${ }^{2}$, Zhang Dayong1,*, He Xiaolan', Guo \\ Shiwei ${ }^{1}$, Peng Chen ${ }^{1}$ and Ma Hongxiang ${ }^{1}$
}

\author{
${ }^{1}$ Jiangsu Key Laboratory for Bioresources of Saline Soils, Provincial Key Laboratory of Agrobiology, Institute of \\ Biotechnology, Jiangsu Academy of Agricultural Sciences, Nanjing 210014, China; ${ }^{2}$ College of life science, Nanjing \\ Agricultural University, Nanjing, 210095, China. \\ *Corresponding author's e-mail: cotton.z@126.com, zhan1658@purdue.edu \\ Huang Yihong and Xu Zhaolong contributed equally.
}

\begin{abstract}
MYB transcription factors in plant play a critical role in regulating response to abiotic stress or adverse environments. In our previous study, DGEP data revealed GmMYB92 is involved in soybean tolerance to abiotic stress. Here, we analyzed the spatial and temporal expression of $G m M Y B 92$ and found $G m M Y B 92$ mainly expressed in roots, young stem and flower tissues during the whole growth stages, and significantly induced by ABA, salt, low temperature $\left(4^{\circ} \mathrm{C}\right)$ and PEG6000 (drought) treatments, and the time points for induction expression varied among different treatments. Bioinformatic analysis of $G m M Y B 92$ promoter region contained many stress-related cis-elements, such as, HSE for heat stress, ARE for anaerobic induction, TC-rich repeat element for defense and stress responsiveness and a CAT-box for specific-expression element in meristem expression. Hereafter, the GmMYB92 promoter region with a 1,951bp DNA fragment including start codon was cloned to drive GUS gene expression to form a chimeric expression cassette used for Arabidopsis Thaliana transformation by means of floral dip. GUS histochemical staining was conducted to characterize the GmMYB92 promoter activity in different tissues including root, stem, leaf, flower and pod from transgenic Arabidopsis Thaliana. The results showed that the gus gene under the control of GmMYB92 promoter mainly expressed in root, young stem and flower, which was consistent with the expression pattern in soybean plant. Our study supply a basis support to further insight into MYB transcription factor's role in the complicated regulation network related to different genes and the mechanism in stress-resistance in plants.
\end{abstract}

Keywords: Glycine max, GmMYB92, promoter analysis, expression, GUS staining.

\section{INTRODUCTION}

Abiotic stress factors, such as salt, drought, high/low temperature cause the world's major crop yield loss of about $50 \%$ annually (http://www.isaaa.org/), especially, the salinization is one of the most important factors causing the global food production reduction (Tuteja et al., 2007; Vij et al., 2007). In plant anti-adversity study, manipulation of single function gene rarely produces reliable stress tolerance, however, modulation or improvement of the activity of a key transcription factor is a more efficient way. Therefore, application of the transcription factor to improve the stress resistance of plants has become one of the research hotspots in recent years (Sun et al., 2009; Liu et al., 2008).

The MYB transcription factor family is one of the most numerous and functionally diverse family in plants (Riechmann et al., 2000; Lipsick et al., 1996). In soybean, $252 M Y B$ genes have been identified so far, accounting for about $4 \%$ of all transcription factors. Among of them, 244 are R2R3-MYB proteins, which can be further grouped into 48 sub-families. Members in each sub-families are functionally conservative. Chromosome distribution model analysis indicates that MYB family are fragmented or tandemrepeated in all 20 chromosomes (Du et al., 2012).

Many studies indicate that MYB transcription factors play important roles in plant secondary metabolism (Uimari and Strommer, 1997; Du et al., 2008), cell differentiation and cycles (Payne et al., 2000; Suo et al., 2003), leaf morphogenesis (Lee and Schiefelbein, 2002; Legay et al., 2007; Yang et al., 2007) as well as the color formation in organ such as pericarp, fruit flesh, leaves and flower (Azuma et al., 2008; Ban et al., 2007; Espley et al., 2007; Takos et al., 2006). MYB transcription factors are also critical for plant hormone response and regulation to environmental stresses (Vannini et al., 2004; Liu et al., 2008, Chen et al., 2003; Hoeren et al., 1998; Lea et al., 2007, Gubler et al., 1997). Increasing evidence suggest the function of $M Y B$ genes in response to abiotic stresses. Yang et al. (2009) treated soybean cultivar Zhong-dou27 with UV-B, drought and salt, and found the GmMYBj6 expression is increased; Gm02g01300 and Gm03g38040 are strongly induced by drought, Gm03g38040 is also significantly enhanced in low temperature and high salt. Gm10g01340 and $\mathrm{Gm} 19 \mathrm{~g} 40650$ are also associated with the plant resistance to high salt, 
drought, and low temperature. In hormone response research, GmMYBJ6 of soybean is induced by $\mathrm{ABA}, \mathrm{GA}_{3}$ and NAA, and the GmMYBJ7 is induced by ABA and NAA (Du et al., 2008). Therefore, plant anti-adversity capability can likely be modulated by controlling the expression of MYB transcription factors (Liao et al., 2008; Miyake et al., 2003; Yang et al., 2009).

GmMYB92, one of the 156 MYB transcription factors genes was identified by Liao (Liao et al., 2008) in soybean in 2008. The gene locates on chromosome 16 and contains 1138bp in full length, with a 256bp intron, and encodes a 293 amino acid peptide. Their research showed that the GmMYB92 can form homodimers or heterodimer with GmMYB76 to produce trans activity. Previously, our lab found that there is the differential expression of GmMYB92 gene between wild and cultivated soybean under salt stress and cloned this gene. After $200 \mathrm{mM}$ $\mathrm{NaCl}$ treatment for two days, GmMYB92 over -expressed composite transgenic soybean plants remained normal while the control seedlings was clearly wilt, indicating GmMYB92 could improve soybean salt-resistance capability (Ali et al., 2012). Moreover, researches about GmMYB92 binding to the promoters of target genes have been reported, but the expression mechanism of GmMYB92 and structure analysis of its promoter have not been reported so far.

In this study, GmMYB92 expression pattern under normal and challenged circumstance were analyzed using semiquantitative RT-PCR. GmMYB92 promoter region with $1,951 \mathrm{bp}$ in length was cloned and used to drive GUS heterologous expression in Arabidopsis thaliana. Multiple elements were identified in the promoter region. The present study provides molecular cues for how to enhance plant tolerance to abiotic tresses by fine regulation of GmMYB92 via plant exogenous hormone, light and temperature in the future.

\section{MATERIALS AND METHODS}

Plant material, bacteria and reagents: Soybean variety Willimas82, Arabidopsis thaliana (Col-0), strains of Escherichia coli DH5 $\alpha$, Agrobacterium tumefaciens EHA105 are preserved in our laboratory. pGEM-T Easy Vector T cloning kit, TaqDNA polymerase PRIME STAR, DNA marker, Trizol reagent, T4 DNA ligase were purchased from Fermentas; reverse transcription kit was from Shanghai Generay Bioengineering Co. Ltd; DNA purification kit was from Promega, the biological reagent was from Shanghai Hao Jia Technology Development Co., ltd respectively.

Total RNA extraction and cDNA synthesis: The samples of roots, stems, leaves from the Soybean Seeding of three-leaf stage, blossom and fruiting period, flowers, pod and the root of seedling of three-leaves stage were treated with $200 \mathrm{mmol}$ $\mathrm{L}^{-1} \mathrm{NaCl}, 20 \%$ PEG6000, $100 \mu \mathrm{mol} \mathrm{L}^{-1}$ ABA solution and $4^{\circ} \mathrm{C}$ for 0 (control), $0.5,3,6$ and $12 \mathrm{~h}$ were collected, grinded in liquid nitrogen, and then total RNA was extracted using a
Plant RNA Kit (Promega, Beijing, China) according to the manufacturer's instructions. Single-stranded cDNA was synthesized using $1 \mu \mathrm{g}$ of total RNA and Oligd (T) 18 primer with the Takara RT-PCR system in a total volume of $20 \mu \mathrm{l}$ according to the instructions.

Gene expression analysis: GmMYB92 Gene expression analysis in different tissues was performed by semi quantitative RT-PCR method using Soybean housekeeping gene GmActin (Glycine max (actin-1-like), Genebank Accession No: XM_003552652) as a control, with $1 \mu \mathrm{l}$ of cDNAs extracted from young root, stems, leaves, flowers and pods respectively in $25 \mu \mathrm{l}$ of PCR mix containing $10 \times \mathrm{PCR}$ buffer $2.5 \mu \mathrm{l}, 10 \mathrm{mmol} \mathrm{L}^{-1} \mathrm{dNTPs} 0.5 \mu \mathrm{L}, 25 \mathrm{mmol} \mathrm{L}^{-1} \mathrm{MgCl}_{2}$ $1.5 \mu$, each primer $1 \mu \mathrm{l}, 5 \mathrm{U} \mathrm{mL}^{-1}$ TaqDNA polymerase 0.2 $\mu$ l. For gene expression analysis in response to different stress, the cDNAs samples from the roots treated with $\mathrm{NaCl}$, PEG6000, low temperature and ABA describe above were used for template. The forward primer sequence of GmActin is 5'-AACAGATGGTCCCTCAT AG-3', and the reverse primer is 5'-TAAATACATTGCCTTCACTC-3'. The primers of GmMYB92 gene are 5'-AACAGATGGTCCCTCATAG3'and 5'-TAAATACATTGCCTTCACTC-3'. PCR reaction was initial at $94^{\circ} \mathrm{C}$ for $3 \mathrm{~min}$, followed by $26 \sim 29$ cycles of 45 seconds denaturation at $94^{\circ} \mathrm{C}, 45$ seconds primer annealing at $55{ }^{\circ} \mathrm{C}$ and 30 s extension at $72{ }^{\circ} \mathrm{C}$, with a final extension at $72^{\circ} \mathrm{C}$ for $10 \mathrm{~min}$, repeated three times. In silico transcript profiling and identification of salt responsive GmMYBs were carried out for detecting further Genome-wide analysis of the MYB transcription factor family in Glycine max. The DGEP data were obtained by developing a rigorous algorithm to detect differentially expressed genes (DEGs) among the $\mathrm{NaCl}$ treated and control samples (Audic and Claverie, 1997).

GmMYB92 promoter cloning and analysis on cis-acting elements: To clone GmMYB92 promoter region, a pair of primers was designed based on the 5' flanking sequence of GmMYB92 from soybean genome online database http://www.phytozome.net/cgi-bin/gbrowse/soybean. The forward primer sequence is 5' AGATTTCAGATTCAATTTCA-3' and the reverse primer sequence is 5'-TTTTTGGCACTCTCACTCAC-3'. Using soybean genome DNA as template, the PCR reaction was carried out in $50 \mu \mathrm{l}$ of system with $10 \times \mathrm{PCR}$ buffer $5 \mu \mathrm{l}, 10$ mmol L ${ }^{-1}$ dNTPs $1 \mu \mathrm{l}, 25 \mathrm{mmol} \mathrm{L}^{-1} \mathrm{MgCl}_{2} 3 \mu \mathrm{l}, 10 \mathrm{mmol} \mathrm{L}^{-1}$

primers $3 \mu \mathrm{l}, 5 \mathrm{U} \mu \mathrm{L}^{-1}$ high fidelity Taq DNA polymerase PRIME STAR $0.5 \mu \mathrm{l}, \mathrm{ddH}_{2} \mathrm{O}$ up to $50 \mu \mathrm{l}$. PCR program was as follows: $98{ }^{\circ} \mathrm{C}$ pre-degeneration for $3 \mathrm{~min}$; followed by 39 cycles of denaturation at $98^{\circ} \mathrm{C}$ for $10 \mathrm{~s}$, primer annealing at $55^{\circ} \mathrm{C}$ for $20 \mathrm{~s}$ and primer extension at $72^{\circ} \mathrm{C}$ for $2 \mathrm{~min}$, with a final extension at $72^{\circ} \mathrm{C}$ for $10 \mathrm{~min}$. The PCR product was cloned into pGEM-T Easy Vector for sequencing. The promoter elements analysis was performed using online tool PLANTCARE (http://bioinformatics.psb.ugent.be/webtools/ plantcare/html/). 
Plasmid construct and plant transformation: To characterize activation of $G m M Y B 92$ promoter in various organs, the 1,951bp promoter fragments from GmMYB92 was cloned into the upstream of the $\beta$-glucuronidase (GUS) reporter gene in the vector pCXGUS-P (Chen et al., 2009) to form a new construct pCXGUS-M92P, which was transformed into Agrobacterium tumefaciens strain EHA105 and further transformed into Arabidopsis thaliana plant by flower dip as described by Clough and Bent (1998).

GUS histochemical staining: Transgenic seeds of Arabidopsis thaliana plants were selected on media containing $50 \mathrm{mg} / \mathrm{L}$ of kanamycin. For $\beta$-glucuronidase (GUS) staining, various tissues from positive lines including root, stem, leaves, flower and pod were incubated in $50 \mathrm{mM}$ sodium phosphate, at a $\mathrm{pH}$ of 7.2, $0.5 \mathrm{mM}$ ferricyanide, 0.5 $\mathrm{mM}$ ferrocyanide, and $2 \mathrm{mM}$ 5-bro-mo-4-chloro-3-indolylbD-glucuronic acid at $37^{\circ} \mathrm{C}$ for $4-6 \mathrm{~h}$ (Jefferson., 1987). Samples were stored in $70 \%$ ethanol before microscopic examination then observed under a microscope (Zeiss Stemi 2000-C, Germany).

\section{RESULTS}

GmMYB92 are mainly expressed in roots, young stems and flowers: To detect the spatial and temporal expression pattern of GmMYB92, semi quantitative RT-PCR method was carried out using different organs in different growth and developmental stages. The results showed that GmMYB92 was highly expressed in root, and the expression levels were maintained at a high level during the whole growing period. High expression levels were also detected in flowers. In the early stem development, GmMYB92 expression was higher in young stem, but very low at flowering and maturity stages, and no expression in leaves and pods (Fig.1).

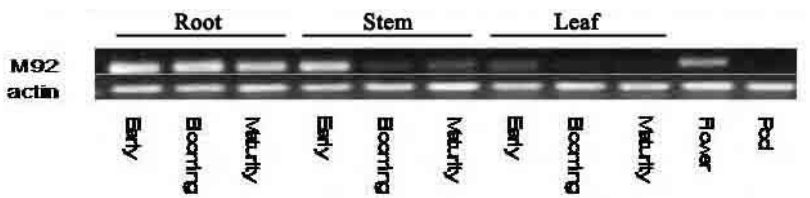

Figure 1. Semi-quantitative RT-PCR analysis of $G m M Y B 92$ expression in different organs and different development stages.

GmMYB92 expression can be induced by $\mathrm{NaCl}, \mathrm{PEG}$ and ABA treatments: GmMYB92 gene expression in the root tissue was also examined with ABA treatment and other treatments, including low temperature, high salt and drought simulation (20\% PEG6000) Compared to the control(no treatment), the expression level of GmMYB92 gene gradually increased and reached the maximum value from $0-1 \mathrm{~h}$ under low temperature treatment $\left(4^{\circ} \mathrm{C}\right)$, but it was not significantly different compared to $\mathrm{NaCl}$, PEG6000 and ABA treatments; The GmMYB92 expression level increased sharply at $3 \mathrm{~h}$, then the expression level decreased slightly at $6 \mathrm{~h}$, but upregulated again at $12 \mathrm{~h}$ after treatment with ABA and PEG6000. The expression of GmMYB92 gene was significantly increased with $200 \mathrm{mM} \mathrm{NaCl}$ treatment for 12 hours and remained high thereafter. Therefore, the expression profiles in response to ABA, low temperature, high salt and drought indicate that GmMYB92 gene is involved in regulation under stresses.

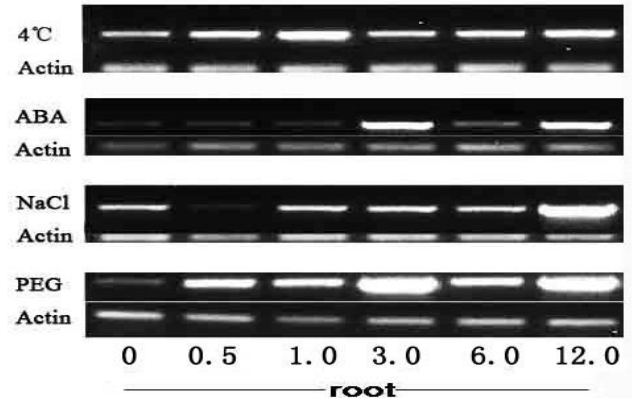

Figure 2. Semi- quantitative RT-PCR analysis of $G m M Y B 92$ in response to $\mathrm{ABA}$ treatment and low temperature, high salt and drought stress.

Difference of $M Y B$ genes expression in Glycine max samples treated with 0 or $200 \mathrm{mM} \mathrm{NaCl}$ were analyzed using soybean Tag sequencing data generated via DGEP. The result showed that a total of $59 \mathrm{GmMYBs}$ with higher expression level were found in Glycine max and $44 M Y B s$ were up-regulated. The expression of $G m M Y B 92$ was 1.77 fold higher than of the control in up-regulated $M Y B s$, which indicated $G m M Y B 92$ is response to $\mathrm{NaCl}$ stress and may be related to enhance salt tolerance of Glycine max.

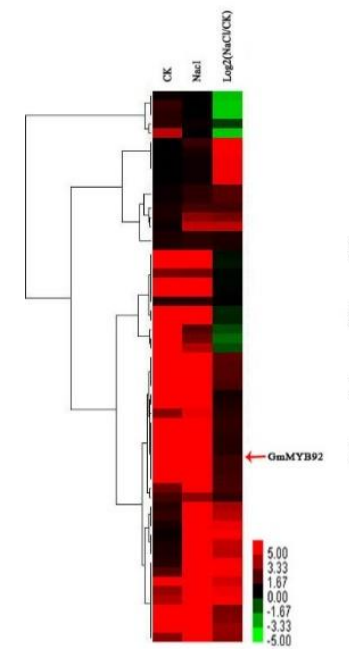

(A)

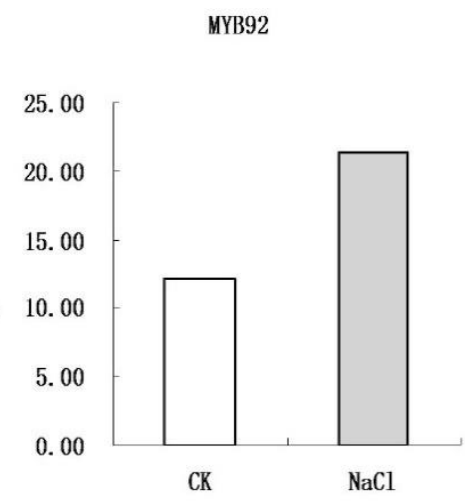

(B)
Figure 3. Expression analysis of $G m M Y B s$ at $12 \mathrm{~h}$ of 200 $\mathrm{mM} \mathrm{NaCl}$ stress in root of trifoliate stage soybean seedlings. (A) Expression profile of 59 GmMYBs based on DGEP data. (B) GmMYB92 expression pattern in high salt stress, the ordinate based on the normalized expression data from DGEP. 
The isolation of GmMYB92 promoter and cis-acting elements analysis:

GmMYB92 promoter region with 1,951 bp in length was cloned by PCR method. The cis acting elements in this promoter were analyzed using an online promoter analysis tool PLANTCARE (Table 1 and Fig. 4). As shown in the

Table1. The cis-acting elements in GmMYB92 promoter.

\begin{tabular}{|c|c|c|c|}
\hline Cis-elements & Number & Position & Function \\
\hline 3-AF3 binding site & 1 & + & part of a conserved DNA module array (CMA3) \\
\hline 5UTR Py-rich stretch & 1 & - & cis-acting element conferring high transcription levels \\
\hline AAGAA-motif & 1 & - & - \\
\hline ARE & 1 & + & cis-acting regulatory element essential for the anaerobic induction \\
\hline AT-rich element & 1 & + & binding site of AT-rich DNA binding protein (ATBP-1) \\
\hline AT1-motif & 1 & - & part of a light responsive module \\
\hline ATGCAAAT motif & 1 & - & cis-acting regulatory element associated to the TGAGTCA motif \\
\hline Box 4 & 1 & + & part of a conserved DNA module involved in light responsiveness \\
\hline Box I & 2 & + & light responsive element \\
\hline CAAT-box & 32 & $+/-$ & common cis-acting element in promoter and enhancer regions \\
\hline CAT-box & 1 & + & cis-acting regulatory element related to meristem expression \\
\hline F-box & 1 & - & - \\
\hline GA-motif & 1 & + & part of a light responsive element \\
\hline GAG-motif & 1 & - & part of a light responsive element \\
\hline GARE-motif & 1 & + & gibberellin-responsive element \\
\hline GATA-motif & 1 & + & part of a light responsive element \\
\hline GT1-motif & 3 & - & light responsive element \\
\hline HSE & 2 & $+/-$ & cis-acting element involved in heat stress responsiveness \\
\hline Skn-1_motif & 2 & + & cis-acting regulatory element required for endosperm expression \\
\hline TATA-box & 132 & $+/-$ & core promoter element around -30 of transcription start \\
\hline TC-rich repeats & 2 & - & cis-acting element involved in defense and stress responsiveness \\
\hline TCT-motif & 2 & + & part of a light responsive element \\
\hline Unnamed_4 4 & 2 & $+/-$ & pl- \\
\hline as-2-box & 1 & - & involved in shoot-specific expression and light responsiveness \\
\hline chs-CMA1a & 1 & - & part of a light responsive element \\
\hline chs-CMA2a & 1 & - & part of a light responsive element \\
\hline circadian & 2 & - & cis-acting regulatory element involved in circadian control \\
\hline
\end{tabular}

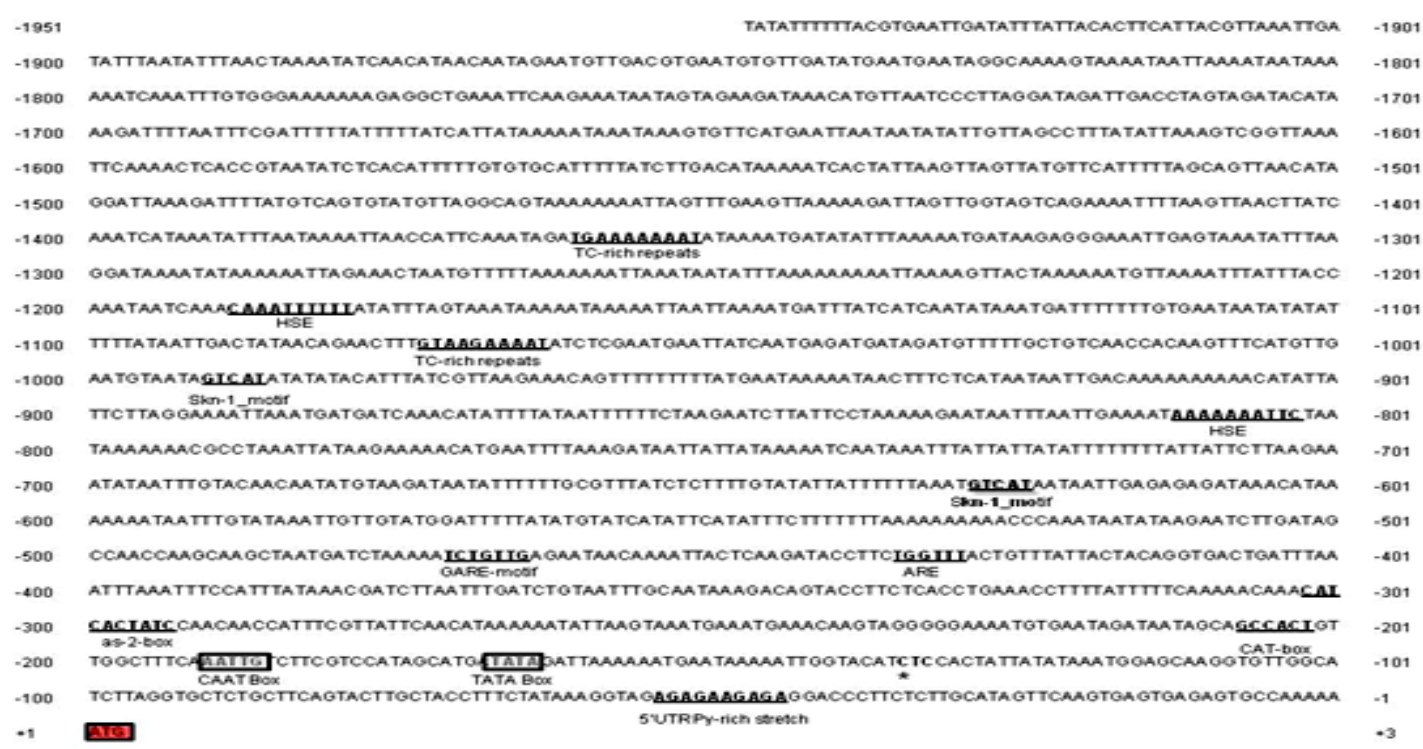

Figure 4. Complete nucleotide sequence of the GmMYB92 promoter region. Nucleotide numbers are indicated to the both sides of each lane. The putative transcriptional start site (asterisk) is indicated as well as the start codon ATG (dark-colored box) of GmMYB92 gene. The TATA box (light-colored box), CAAT box (light-colored box) and other main cis-elements (underlined and bold) are also showed. 


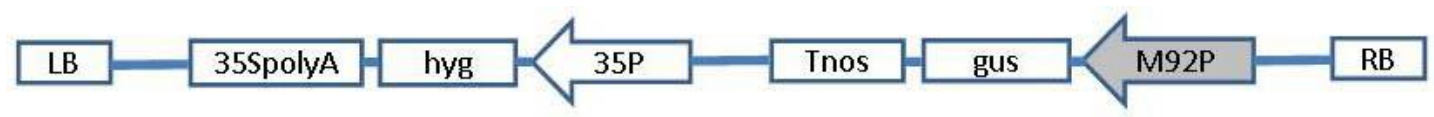

Figure 5. Schematic structure of the plant expression vector pCXGUS-M92P generated by insertion of GmMYB92 promoter fragment (M92P).

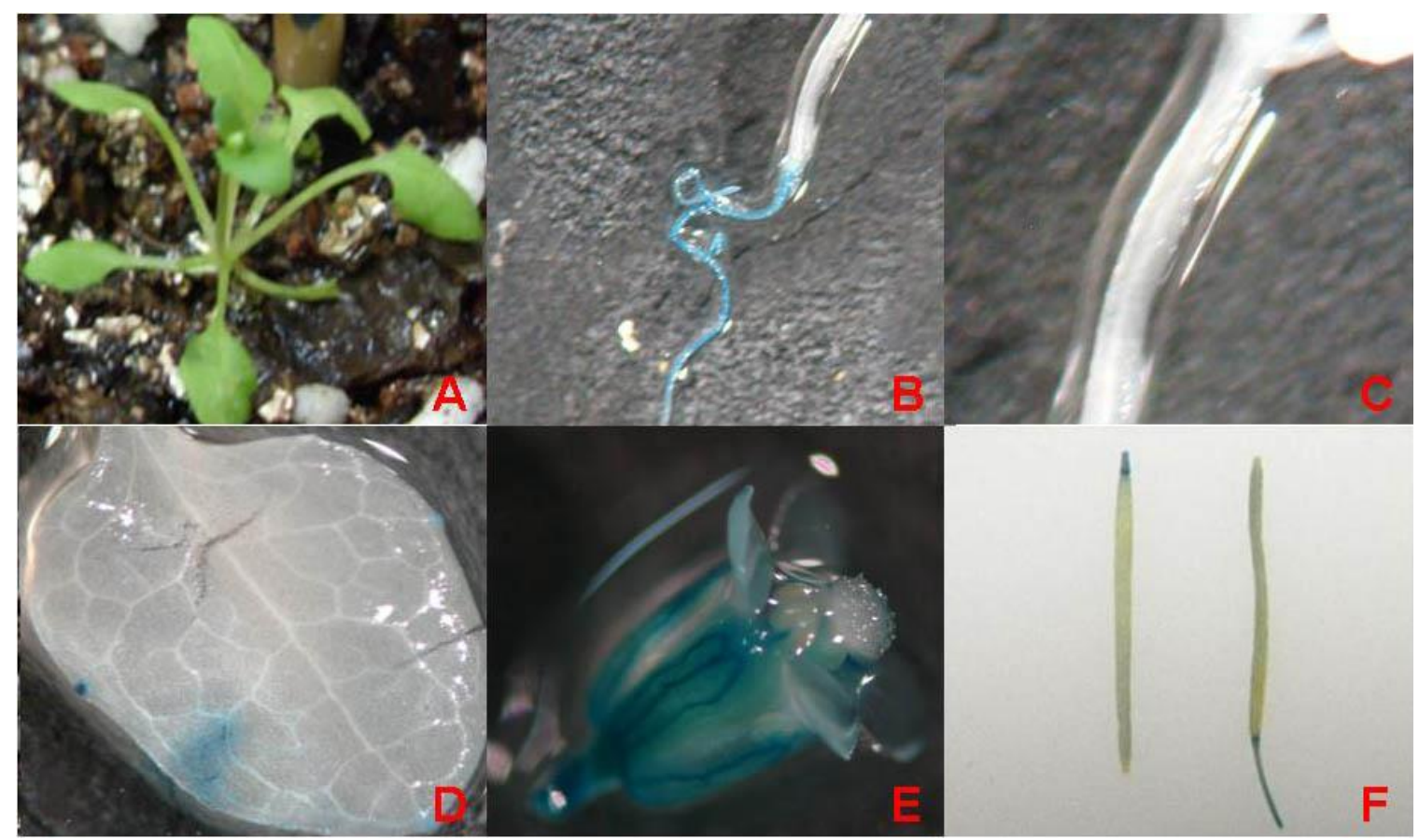

Figure 6. GUS staining in various tissues from transgenic Arabidopsis thaliana harboring GUS gene driven by GmMYB92 promoter. A: 3-week-old transgenic seedling; B: root; C: stem; D: leaf of 3-week-old seedling; E: flower at blooming stage; F: pods of transgenic Arabidopsis thaliana.

table, it contains five types of cis acting elements including transcription-related, light reaction, tissue specific expression, abiotic stress response and hormone response elements respectively. Transcription-related components often contain, in addition to normal promoter, TATA frame, CAAT frame, 5UTR Py-rich stretch elements. Total 15 Light response elements are exist in the area, belonging to 11 groups. There are three tissue specific expression elements, CAT box for meristem tissue, Skn-1 for endosperm and as-2box for bud, respectively. Stress response elements with heat shock element HSE, hypoxia inducible element ARE and the stress response and defense element TC-rich repeat are contained. GARE, the gibberellic acid response element, is also. These elements are distributed in both sense and antisense strands. Among them, the CAAT box, TATA box and light responsive elements have multiple copies, while others only have one or two copies.

GmMYB92 promoter drove GUS expression analysis in transgenic Arabidopsis thaliana: Reporter expression cassette was constructed by fusing the GmMYB92 promoter and GUS gene (Fig.5), and transformed into Arabidopsis thaliana using the flower dip method. GUS histochemical staining were carried out in transgenic seedlings of $\mathrm{T} 1$ generation. The results showed that high GUS expression was found in the root and flower. No GUS expression was found in stems and pod (Fig.6), which was consistent with the organ expression pattern in soybean seedling.

\section{DISCUSSION}

The promoters of different stress-related gene often contain the same cis acting elements, being regulated in a similar way or through similar molecular mechanism. In other words, the transcriptional regulatory gene can regulate the expression level of a series of stress related genes, thereby enhance the resistance capability to the stress (Liu et al., 2008). Through the analysis of the Arabidopsis thaliana drought, high salt and $\mathrm{ABA}$ inducible promoter region of genes, TAACTG, the core sequence for MYB binding was identified (Urao et al., 1993). MYB transcription factor can also bind with MYBS I (conservative sequence T/CAACG/TGA/C/TA/C/T) , MYBS II , ( conservative sequence TAACTAAC), CNGTTR and GKTWGTTRGKTWGGTR (N : A, G, C or 
T; K : G or T; R : A or G; W : A or T), (Romero et al., 1998). Liao et al. (2008) showed that GmMYB92 can recognize and bind MBSI recognition sequence (TATAACGGTTTTTT), MRE4 (TCTCACCTACC) and Mmre1 (CCGGAAAAAAGGAT). Plant stress tolerance related genes such as DREB2A, RD17, RD29A, RD29B, P5CS, COR66 and COR78 are downstream genes of MYB transcription factor. In $G m M Y B 92$ transgenic Arabidopsis, DREB2A, RD17 and P5CS are increased while COR66 and COR78/rd29A expression level remain low, suggesting that GmMYB92 regulation on the downstream target genes is rather complicated. It may, alone or synergistically with other transcription factors, up-regulate or down-regulate target gene transcription level, eventually improves the stress tolerance of plants.

The interaction among genes forms a complex gene regulatory network in cell. While MYB transcription factor regulate target gene expression, its own activity is regulated by other proteins at all levels, in another word, it is the intermediate target of other regulatory factors (Gonzalez et al., 2008). RNA and protein are two kinds of molecules regulate the expression of transcription factor $M Y B$ gene. $M Y B$ gene is a common target of small RNAs (miRNAs) and trans silencing of RNAs (ta-siRNAs). For instance, miR159 acts on AtMYB33, AtMYB35, in AtMYB65 and AtMYB101, and thus regulates anther and pollen development (Gonzalez et al., 2008). In the MADS family, embryo development related AGL15 protein can bind 29 different $M Y B$ genes (Zheng et al., 2009). In addition to proteins, these genes directly regulate MYB transcription factor. For example, AtMYB123, AtMYB2, AtMYB66, AtMYB0 and AtMYB33 are direct regulatory sites for up to 552 genes. The regulation process also involves bHLH chaperone proteins (KoshinoKimura et al., 2005). In general, MYB transcription factors can be divided into two categories. One is the ABA related transcription factors, promoter of many ABA induced genes contain ABRE consensus sequence. The other is ABA independent MYB transcription factors. Their promoter region contains CRT/DRE or other elements, acting as MYB protein recognition sites, and thus regulated by the corresponding factors (Liu et al., 2008). GmMYB92 promoter contain neither ABRE, nor CRT/DRE cis acting element, but in state of gibberellic acid response element GARE, suggesting that it belongs to ABA independent MYB transcription factors, and is related to gibberellic acid signaling pathway. Sequence analysis shows GmMYB92 promoter region contains the thermal stress components HSE, anaerobic stress inducible elements ARE, and indicating that the gene is involved in plant stress responses. In addition, the promoter region also contains multiple light response elements, some other elements that are rarely found in other $M Y B$ promoters, including 3 '-AF3, AT rich element binding site, AT motif, F-box and chs-CMA2a etc. The roles of these elements in the regulation of GmMYB92 transcription are not clear so far. Further experimental studies are required to understand their functions.

$M Y B$ gene family has the differential temporal and spatial expression patterns. For example, AtMYB33 and AtMYB65 genes in Arabidopsis thaliana express in a variety of organs and tissues (Gocal et al., 2001). C1 and Pl in corn has a role in the regulation of anthocyanin synthesis, but $\mathrm{C} 1$ is only expressed in the aleurone layer and flower organs, while Pl is restricted in vegetative tissues (Piaaza et al., 2002). Northern blot results show that, GmMYBJ6 expression is only detected in the leaves, suggesting tissue specific expression of GmMYBJ6 (Yang et al., 2009). In this study, the GmMYB92 gene expression and its promoter driving GUS expression in Arabidopsis show that the expression in roots and young stems, leaves and flowers is high, but is low in fully developed response tissues and organs. We suspected that the stable, high level expression is conducive to its ability to regulate the stress responsive genes in order to improve the resistance ability of plants. Expression in young tissues may be related to existence of Meristem specific expression element CAT box, suggesting GmMYB92 plays a role in root, stem growth and flower development and tolerance to stresses.

Acknowledgments: This study was sponsored by the National Science Foundation of China (31101166); National Science Foundation of Jangsu Province (BK20151364); Jiangsu Key Laboratory for Bioresources of Saline Soils(JKLBS2012002), the Special Fund for Independent Innovation of Agricultural Science and Technology in Jiangsu CX(12)1005-2.

\section{REFERENCES}

Ali, Z., D.Y. Zhang, Z.L. Xu, L. Xu, J.X. Yi, X.L. He, Y.H. Huang, X.Q. Liu, A.A. Khan, R.M. Trethowan and H.X. Ma. 2012. Uncovering the salt response of soybean by unraveling its wild and cultivated functional genomes using tag sequencing. Plos One. 7:e48819.

Audic, S. and M. Claverie. 1997. The significance of digital gene expression profiles. Genome Res. 7:986-995.

Azuma, A., S. Kobayashi, N. Mitani, M. Shiraishi, M. Yamada, T. Ueno, A. Kono, H. Yakushiji and Y. Koshita. 2008. Genomic and genetic analysis of $M Y B$-related genes that regulate anthocyanin biosynthesis in grape berry skin. Theor. Appl. Genet. 117:1009-1019.

Ban, Y., C. Honda, Y. Hatsuyama, M. Igarashi, H. Bessho and T. Moriguchi. 2007. Isolation and functional analysis of a MYB transcription factor gene that is a key regulator for the development of red coloration in apple skin. Plant Cell Physiol. 48:958-970.

Chen, S.B., P. Songkumarn, J.L. Liu and G.L. Wang. 2009. A versatile zero background T-vector system for gene cloning and functional genomics. Plant Physiol. 150:1111-1121.

Chen, S.C., S.Q. Peng, G.X. Huang, K.X. Wu, X.H. Fu and 
Z.Q. Chen. 2003. Association of decreased expression of a MYB transcription factor with the TPD (tapping panel dryness) syndrome in Hevea brasiliensis. Plant Mol. Biol. 51:51-58.

Clough, S.J. and A.F. Bent. 1998. Floral dip: a simplified method for Agrobacterium- mediated transformation of Arabidopsis thaliana. Plant J. 16:735-743.

Du, H., S.S. Yang, Z. Liang, B.R. Feng, L. Liu, Y.B. Huang and Y.X. Tang. 2012. Genome-wide analysis of the MYB transcription factor superfamily in soybean. BMC Plant Biol. 12:1-22.

Du, H., W.J. Yang, L. Liu, X.F. Tang, Y.M. Wu, Y.B. Huang and Y.X. Tang. 2008. Cloning and functional identification of the two MYB transcription factors GmMYBJ6 and GmMYBJ7 in soybean. Acta Agron. Sin. 34:1179-1187.

Espley, R.V., R.P. Hellens, J. Putterill, D.E. Stevenson, S. Kutty-Amma and A.C. Allan. 2007. Red colouration in apple fruit is due to the activity of the MYB transcription factor, MdMYB10. Plant J. 49:414-427.

Gocal, G.F.W., C.C. Sheldon, F. Gubler, T. Moritz, D.J. Bagnall, C.P. MacMillan, S.F. Li and R.W. Parish. 2001. $G A M Y B$-like genes, flowering and gibberellin signaling in Arabidopsis. Plant Physiol. 127:1682-1693.

Gonzalez, A., M. ZHAO, J.M. LEAVITT and A.M. Lioyd. 2008. Regulation of the anthocyanin biosynthetic pathway by the TTG1/bHLH/MYB transcriptional complex in Arabidopsis seedlings. Plant J. 53:814-827.

Gubler, F., R.J. Watts, R. Kalla, P. Matthews, M. Keys and J.V. Jacobsen. 1997. Cloning of a rice cDNA encoding a transcription factor homologous to barley GAMYB. Plant Cell Physiol. 38:362-365.

Hoeren, F.U., R. Dolferus, Y.R. Wu, W.J. Peacock and E.S. Dennis. 1998. Evidence for a role for AtMYB2 in the induction of the Arabidopsis alcohol dehydrogenase gene (ADH1) by low oxygen. Genetics 149:479-490.

Jefferson, R.A. 1987. Assaying chimeric genes in plants: the GUS gene fusion system. Plant Mol. Biol. Rep. 5:384405.

Koshino-Kimura, Y., T. Wada, T. Tachibana, R. Tsugeki, S. Ishiguro and K. Okada. 2005. Regulation of CAPRICE transcription by MYB proteins for root epidermis differentiation in Arabidopsis. Plant Cell Physiol. 46:817-826.

Lea, U.S., R. Slimestad, P. Smedvig and C. Lillo. 2007. Nitrogen deficiency enhances expression of specific MYB and bHLH transcription factors and accumulation of end products in the flavonoid pathway. Planta 225:1245-1253

Lee, M.M. and J. Schiefelbein. 2002. Cell pattern in the Arabidopsis root epidermis determined by lateral inhibition with feedback. Plant Cell 14:611-618.

Legay, S., E. Lacombe, M. Goicoechea, C. Brière, A. Séguin, J. Mackay and J. Grima-Pettenati. 2007. Molecular characterization of EgMYB1, a putative transcriptional repressor of the lignin biosynthetic pathway. Plant Sci. 173:542-549.

Liao, Y., H.F. Zou, H.W. Wang, W.K. Zhang, B. Ma, J.S. Zhang and S.Y. Chen. 2008. Soybean GmMYB76, GmMYB92, and GmMYB177 genes cofer stress tolerance in transgenic Arabidopsis plant. Cell Res. 18:1047-1060.

Lipsick, J.S. 1996. One billion years of MYB. Oncogene 13:223-235.

Liu, L., H. Du, X.F. Tang, Y.M. Wu, Y.B. Huang and Y.X. Tang. 2008. The roles of MYB transcription factors on plant defense responses and its molecular mechanism. Hereditas 30:1265-1271.

Miyake, K., T. Ito and M. Sends. 2003. Isolation of a subfamily of genes for R2R3-MYB transcription factors showing up-regulated expression under nitrogen nutrient-limited conditions. Plant Mol. Biol. 53:237-245.

Payne, C.T., F. Zhang and A.M. Lloyd. 2000. GL3 encodes a bHLH protein that regulates trichome development in Arabidopsis through interaction with GL1 and TTG1. Genetics 156:1349-1362.

Piaaza, P., A. Procissi, G.I. Jenkins and C. Tonelli. 2002. Members of the $\mathrm{c} 1 / \mathrm{pl}$ regulatory gene family mediate the response of maize aleurone and mesocotyl to different light qualities and cytokinins. Plant Physiol. 128:10771086.

Riechmann, J.L., J. Heard, G. Martin, L. Reuber, C. Jiang, J. Keddie, L. Adam, O. Pineda, O.J. Ratcliffe, R.R. Samaha, R. Creelman, M. Pilgrim, P. Broun, J.Z. Zhang, D. Ghandehari, B.K. Sherman and G. Yu. 2000. Arabidopsis transcription factors: genome-wide comparative analysis among Eukaryotes. Sci. 290:21052110 .

Romero, I., A. Fuertes, M.J. Benito, J.M. Malpica, A. Leyva and J. Paz-Ares. 1998. More than 80R2R3-MYB regulatory genes in the genome of Arabidopsis thaliana. Plant J. 14:273-284.

Sun, X., J.Y. Liu, X.H. Yuan, X.W. Pan, W.G. Du, H.X. Ren, Y.B. Ma, A.B.E. Jun, L.J. Qiu and B.H. Liu. 2009. Cloning and expression analysis of $G m M Y B$ genes induced by abiotic stresses. Acta Agron. Sin. 38:360-368.

Suo, J., X. Liang, L. Pu, Y. Zhang and Y. Xue. 2003. Identification of GhMYB109 encoding a R2R3 MYB transcription factor that expressed specifically in fiber initials and elongating fibers of cotton (Gossypium hirsutum L.). Biochem. Biophys. Acta 1630:25-34.

Takos, A.M., F.W. Jaffe, S.R. Jacob, J. Bogs, S.P. Robinson and A.R. Walker. 2006. Light-induced expression of a $M Y B$ gene regulates anthocyanin biosynthesis in red apples. Plant Physiol. 142:1216-1232.

Tuteja, N. 2007. Mechanisms of high salinity tolerance in plants. Methods Enzymol. 428:419-438.

Uimari, A. and J. Strommer. 1997. MYB26: a MYB-like protein of pea flowers with affinity for promoters of 
phenylpropanoid genes. Plant J. 12: 1273-1284.

Urao, T., K. Yamaguchi-Shinozaki, S. Urao and K. Shinozaki. 1993. An Arabidopsis MYB homolog is induced by dehydration stress and its gene product binds to the conserved MYB recognition sequence. Plant Cell 5:1529-1539.

Vannini, C., F. Locatelli, M. Bracale, E. Magnani, M. Marsoni, M. Osnato, M. Mattana, E. Baldoni and I. Coraggio. 2004. Overexpression of the rice OsMYB4 gene increases chilling and freezing tolerance of Arabidopsis thaliana plants. Plant J. 37:115-127.

Vij, S and A.K. Tyagi. 2007. Emerging trends in the functional genomics of the abiotic stress response in crop plants. Plant Biotech. J. 5:361-380.

Yang, W.J., Y.M. Wu and Y.X. Tang. 2009. Expression and functional analysis of GmMYBJ6 from soybean. Hereditas 31:645-653.

Yang, X.Y., J.G. Li, M. Pei, H. Gu, Z.L. Chen and L.J. Qu. 2007. Over-expression of a flower-specific transcription factor gene AtMYB24 causes aberrant anther development. Plant Cell Rep. 26:219-228.

Zheng, Y., N. Ren, H. Wang, A.J. Stromberg and S.E. Perry. 2009. Global identification of targets of the Arabidopsis MADS domain protein AGAMOUS-Like15. Plant Cell 21:2563 -2577. 